\title{
Architecture of STL Model of New Communication Network
}

\author{
Aibao Wang ${ }^{1,2}$ and Guangzhao Zhang ${ }^{1}$ \\ ${ }^{1}$ Zhongshan University \\ ${ }^{2}$ China Telecom \\ wangab@chinatelecom.com.cn, isszgz@mail.sysu.edu.cn
}

\begin{abstract}
New communication network taking Internet as representative is being used extensively, but traditional communication theory cannot give good explanations and instructions on these new communication networks. Taking anthropotomy as a reference model, we analyze the systematic integration and functional requirements of new communication network with Internet as its representative in this paper. Further, we put forward the STL model of new communication network. Through the illustration for STL model, we have new knowledge on the Internet-representative communication network, which has certain use for the development of related theory and technologies.
\end{abstract}

Keywords: Communication theory, network architecture, next generation network.

\section{Introduction}

Tracing back the development of communication technology, it has been more than 20 years since the digital technology acquired its application in communication field, especially the fast development of satellite communications, fiber optical communications, and mobile communications made communication much more easily in people's daily work and life. However, comparing with the extensive application and involvement in social life, the development of communication theory appears to be standing still for relevant communication theory cannot keep up with the development of communication technology.

As for the definition of communication, generally speaking, the traditional one is "the process of realizing the effective message transferring (or exchanging) from one site to another (or multi-site), using technologies like electromagnetism, photoelectricity, etc." But in recent years, the development of communication technology, especially the development of Internet and mobile communication technologies, has made the definition of communication in reality greatly surpass the definition of communication in communication theory.

Firstly, the purpose of communication is not only to transmit certain message, but also to take on more extensive bound, for example, telephone chat, joke transmitted by short messages, Internet games, and current popular BLOG. Strictly speaking, they are all kinds of communication, but the purpose of communication is entertainment or for the need of personal self-express. 
Secondly, seeing from the obstacle communication can surmount, traditional communication only pays attention to the area crossing communication skills, but does not take two obstacles of spanning time and language into consideration; but seeing from the application of existing Internet, surmounting two obstacles of time and language has been possible. For example, EMAIL, which surmounts the time limit and realizes the intercommunication between two nodes at EMAIL sending and receiving time; and Text-To-Speech (TTS) technology based communication between text media and speech media has realized the communication between different languages. What's more, with the development of Internet technology and application, the expressing method has taken on diverse trends. Speech communication is no more the only way for people's daily communication. Text communication (message communicating and chat on-line), and video communication began to involve in people's daily life.

Therefore, the traditional definition of communication can not cover the ability possessed by real communication anymore. In order to instruct the development of communication technology and application effectively, communication science is in dire need of reknowing and redefining the definition of communication.

Since the appearance of Internet, especially the popular applications such as: WEB/Email/IM/BLOG, etc., people have realized that the Internet is in fact a communication network which can provide more abilities. The open character of Internet makes everyone have the opportunity to participate in the creation for all kind of contents and applications; and the abundant computing abilities together with the great capacity memory power promotes the Internet to explode vast intelligence application, which can be independent of people's intervene to Auto-Run. Therefore, Internet-representative new communication network is no longer a simple communication system, but a smart and elaborative intelligent processing system, (for convenience, new communication network refers to Internet-representative new generation network in this paper), its service object does not limit to the speech among people, but in a greater degree involves to all layers of social life. The intelligence of new communication network mainly presents the following aspects.

Taking anthropotomy as reference model, the purpose of this paper is to analyze the systematic integration and functional requirements of new communication network with Internet as its representative, and put forward the STL model and its framework consideration. Through the illustration for STL model, we have a whole new knowledge on Internet-representative new generation communication network, which has certain use for the development of relative theory and technology.

\section{Related Work}

Research on network communication principle and architecture has been the hotspot of academe for a long time. The famous expert in computer network D. Clark gave the object for development of Internet and main design principle based on "Edge Theory" in [1], which established basic architecture and developing direction for the Internet. In 1980s, standardization has been the masterstroke for the development of network theory and technology.

Internet is a successful example of communication network, but currently, people still cannot establish a theory model for complex and giant system as Internet. Because 
of the continuous development of computer's traffic type, traditional mathematic model of network behavior such as Markov modulated Poisson Process and so on cannot reflect the real network behavior anymore. In recent years, relative researches domestically or abroad are all trying out new mathematic model reflecting certain real features of network, such as self-similarity and long-range dependence model, etc., through the analysis and research on a great deal traffic data. But due to the high burst and randomcity of modern network information transmission, up to now, satisfactory theory and model which can reflect network statement and behavior from all aspects are not found yet.

With people's increasing need for the Internet, many new thought and technology are introduced to Internet-relative theories and practices. Existing IPv4 protocol has the problem of inadequate address space. In order to provide more address space, IPv6 protocol became the IETF warmly recommended network layer protocol of new generation Internet [3]. IPv6 has integrated the IPSec security protocol, which can solve the problem of network security to some extend. Now, America, Europe Union, and Japan are all vigorously supporting the construction of IPv6 backbone network. IPv6 network already or being constructed include CERNET2 of our country, Abilene [4] of America, GEANT [5] of Europe Union, and APAN [6] of Asia-Pacific, etc.

Researchers have put forward a lot of new Internet architecture, such as NGI [7], NewArch [8-10], FIND [11], GENI [12], NSFCNET [13], etc. They are trying to design a new Internet which can better satisfy people's need in network communication. There are also some proposals to improve the current Internet architecture, such as IPNL [14], ROFL [15], NIRA [16], I3 [17], TRIAD [18] and etc. However, up to now, no researchers give better explanations and instructions on Internet-representative new communication network from the perspective of communication theory, which is also the main research purpose of this paper.

\section{New Communication Network System}

New communication network is a smart intelligence system and what kind of components and functions the intelligent system should possess? Now, we take anthropotomy as model theory of new communication network system, and take new communication as a "Human Body System" to anatomize, analyze, and research.

Before analyzing the new communication network system, let us first have a look at the components of human body. From the perspective of anthropotomy, human body consists of skeleton, muscle, nerve, cerebra, heart, blood vessel, and all other kinds of internal organs. Human as a special animal, its purpose of existence is fundamentally the same with other animals, which is "act", but people endow "act" with more meanings (e.g. labor, entertainment, sport, travel, etc). The mainly basic apparatus in human body system which realize "act" are muscle, skeleton and nerves. Therefore, view from the 'act' function, muscle, skeleton and nerves are the fundamental components of the human body system. And all other organs work together as the human body support-system. As a normal man, in order to maintain the 'act' function work well, his support-system such as heart, lungs etc. must be in powerful status.

When we take new communication as an intelligent "human body system" to analyze, the new communication system consist of following parts: network 
architecture(signal transmission subsystem), information storage module, information expression module, resource management subsystem, power supply subsystem, application servers, operation supporting subsystem, security protection subsystem, etc. As new communication network taking "communication" as its basic function, its three basic parts are: network infrastructure, storage module, and information expressing module. Among them, network infrastructure refers to circuit transmission and switching system such as communication termination, access network, backbone network, and core network. Storage module includes not only independent information storage subsystem, but also attached storage equipment in network and termination equipments. And information expressing module mainly refers to all kinds of communication terminations, it also include internal software parts of equipment in network nodes for information recognizing and switching. possess the various information expressions and switching abilities from different languages.

As for other parts in the new communication network system, which are similar to the various supporting organs in human body system, belong to OSS(Operation Support System) which serve for the fundamental function of main body in communication system to help and make sure the network can provide 'communication services' with abundant contents continuously and smoothly.

So, from the viewpoint of 'human body system', the new communication network system should have:

- An architecture which realize the fundamental function of the network system. But the fundamental function 'Communication' now have more meaning than the traditional definition. More detailed about the architecture meaning will show in chapter4.

- An OSS which should run effectively and healthy in line with the architecture of the main body of the new communication network in order to guarantee the total network system run well.

\section{STL Model Design Adapted to New Communication Network System}

We may see from above, the new communication network realized communication content has surpassed the defined traditional communication in existing communication theory; at the same time, from the anatomy point of view, current Internet still cannot completely possess the function and corresponding components which new communication expected, for example, security and antivirus subsystem, resource operation and management subsystem, etc.

This has brought forward such a proposition for us: how should new communication network infrastructure be like? How should existing communication system theory evolve to guide the need for the continuing development of new communication network?

Trying to answer the above questions, we put forward the STL model of new communication network infrastructure on the basis of analyzing three direct correlative abilities in accomplishing new communication function, namely, network infrastructure, information storage unit, language expressing and switching unit. 


\subsection{STL Model for New Communication}

We know from the function analysis needed in new communication network of the previous chapter that refresh understanding new communication network need surmount not only the geographic limitation (existing communication only consider the problem of space domain, and realizing the communication among users in different geographic places), but also the limitation of time domain and language domain, realizing the communication among communication bodies of different places, different time and different language expressions(Fig. 1). On mathematic model, we can take it as a three-domain (three variables) communication model, taking the three initials of words SPACE, TIME, LANGUAGE; we call the new communication network infrastructure model as STL model.

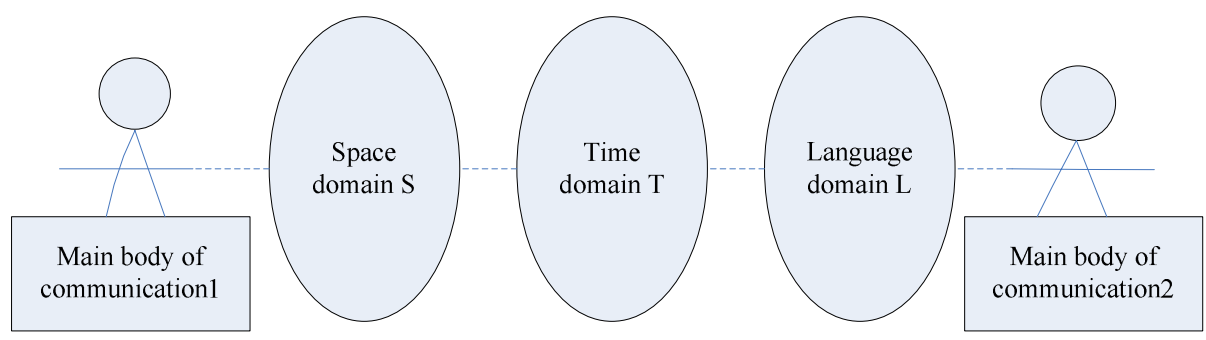

Fig. 1. Illustration of STL model of new communication network

The relationship of three-domain variables needed by communication network in fig. 1 is serial interconnection, which is consistent with the space domain, time domain, and language domain where any communication process will come through in reality. In the fig.1, space-domain as "body (network infrastructure)" mainly undertakes the function of multicast delivery and multicast switching; time-domain as information storage section is mainly responsible for the information transmission on time latitude; and language-domain as the information expressing and computing section, is mainly responsible for the semantics recognition, expression and translation.

Because two latitudes are added on the basis of space domain, comparing with the traditional communication network, there will be a substantial jump change on the function of the new communication network. For example, after adding the time-domain, new communication network can realize the communication between present and future or past; after adding the language-domain, new communication network can not only realize the communication and conversation among different language group, but also realize the communication between people and machine, and even people and animals or plants.

Then we begin the analysis on the output result of several combine scenes of STL model in mathematic perspective.

Space-domain can be denoted as vector $S, S=[S(1), S(2), \ldots, S(k), \ldots, S(n)]$, among which, $\mathrm{k}(1,2, \ldots \mathrm{n})$ denotes the different geographic positions in the space and $\mathrm{S}(\mathrm{k})$ we called it position factor corresponding the output intensity of signal, for convenience, under default statement, $\mathrm{S}(\mathrm{k})=1(\mathrm{k}=1, \ldots \mathrm{n})$. 
Time-domain can be denoted as variable $\mathrm{T}, \mathrm{T}=\mathrm{T}(\mathrm{k})$, denoting time delay when in the position of $\mathrm{k}$ (time factor for short);

Language domain is denoted as variable $\mathrm{L}, \mathrm{L}=\mathrm{L}(\mathrm{k})$, denoting the language expression when in the position $\mathrm{k}$ (semantic factor);

Now we suppose a message $\mathrm{x}$ was input into the communication system, considering a communication process must pass through the three domains of communication network, namely, space domain, time domain, and language domain, thus the output message $\mathrm{Y}$ is:

$$
\begin{aligned}
& Y=S \cdot T \cdot L \cdot x \\
& =[S(1) \cdot T(1) \cdot L(1), S(2) \cdot T(2) \cdot L(2), \ldots, S(k) \cdot T(k) \cdot L(k) \ldots, S(n) \cdot T(n) \cdot L(n)] \cdot x
\end{aligned}
$$

After a message $x$ enters the STL model network, there will be many output messages, the finite time, geographic position, and semantic expression used can all be different. Following are eight possible message output scenes.

\section{1) Point-to-point real-time communication mode, adopting the same language expression.}

At this time, $\mathrm{n}=1, \mathrm{~S}(1)=1, \mathrm{~T}(1)=1$ (no time delay), $\mathrm{L}(1)=1$ (the same language). Thus $Y=S$ (1).T (1).L (1) =x, i.e. the message output end acquired is consistent with the input end. Real scene corresponds to the point-to-point real-time communication mode like PSTN, instant messenge, etc. In these scenes, both sides have the on-line real-time communication with each other, adopting the same language.

\section{2) Point-to-point real-time communication mode with output and input adopt- ing different language expressions}

At this time, output $\mathrm{Y}=\mathrm{L}(1)$.x. i.e. output message is the product of original language message and semantic factor L(1). Real scene corresponds to phone-calling or text chating existing Synchronized Translation or Text-To-Speech function.

\section{3) Point-to-point non-real-time communication mode, adopting the same lan- guage.}

At this time, output $\mathrm{Y}=\mathrm{T}$ (1).x. i.e. output message is the product of original language message and time factor $\mathrm{T}$ (1). Real scene corresponds to communication mode adopting EMAIL sending process. The message from sender will be received by the accepter after time $\mathrm{T}(1)$.

\section{4) Point-to-point non-real-time communication mode with output and input adopting different language expressions.}

At this time, output $\mathrm{Y}=\mathrm{T}$ (1).L (1).x, i.e. output message is the product of original language message, time factor $\mathrm{T}$ (1), and semantic factor L (1). Real scene is similar to WEB visiting, which is when the visitor acquired the information of website; auto translation software will switch its webpage content to languages or characters he or she can understand.

\section{5) Point-to-multipoint real-time communication mode, adopting the same lan- guage expression.}

Thus, the output of multi-position point is: $\mathrm{Y} 1=\mathrm{X}, \mathrm{Y} 2=\mathrm{X}, \ldots, \mathrm{Yn}=\mathrm{X}$. Real scene corresponds to the conference meeting scene where people communication by the same language. 
6) Point-to-multipoint real-time communication mode with input and outputs adopting different language expressions.

At this time, $\mathrm{Y} 1=\mathrm{L}(1) . \mathrm{x} ; \mathrm{Y} 2=\mathrm{L}(2) . \mathrm{x} ; \ldots ; \mathrm{YN}=\mathrm{L}(\mathrm{n}) . \mathrm{x}$. Real scene corresponds to the conference meeting scene where listeners need Synchronized Translation to understand the lecturer.

7) Point-to-point non-real-time communication mode, adopting the same language expression.

Thus, the output of multi-position point is: $\mathrm{Y} 1=\mathrm{T}(1) . \mathrm{x} ; \mathrm{Y} 2=\mathrm{T}(2) . \mathrm{x} ; \ldots ; \mathrm{YN}=\mathrm{T}$ (n).x. Real scene corresponds to EMAIL send to group-accepter or WEB visited by multi-people, the reading time is delayed differently for each accepter or visitor.

8) Point-to-multipoint non-real-time communication mode with input and outputs adopting different language expressions.

Thus, the output of multi-position point is: Y1=T (1).L (1).x; Y2=T (2).L (2).x; ...; $\mathrm{YN}=\mathrm{T}(\mathrm{n}) . \mathrm{L}(\mathrm{n}) . \mathrm{x}$. Real scene corresponds to webpage visiting of WEB mode (the webpage support the translation function among multi-language), the website visiting finite-time of everyone is different, and the semantic may be different, too.

\subsection{Consideration of the STL Mode Based Network Architecture}

Limited by the paper volume, the solution of the STL mode will not be revealed in this paper. Here we give some consideration of the solution when we do the STL network architecture design.

1) the objective of the STL mode is to provide a communication theory or mode which can explain the Internet representative communication network, and from the STL mode, we hope to find more value(good business mode) and more application from the new network.

2) STL mode based network not only should realize the 3-domain communication functions, but also should provide a perfect OSS. By the OSS, the STL network will not only solve the QOS and Security issues which are the hot topic in the current days, but also, will give the STL network intelligence, reliable and continuous developmental functions etc.

3) STL network should run on the top of IP4 base Internet while evolve a intelligent terminal driver. Among them, the solution for the space-domain architecture will refer to the principle of the Content Switch Network architecture[19]. And the architecture for time-domain and Language-domain will require new design.

\section{Conclusion}

The Internet has contributed a lot to human-being since its first appearance, but the traditional communication theory cannot give a satisfactory explanation to the Internet-representative new communication network. This paper analyzes the systematic components and function requirement of Internet-representative communication network, and on the basis of which brings forward a STL model and its interface of new communication network. Through the description of STL model, we have a whole new knowledge of Internet-representative new communication network, which is helpful to the development of communication science and relative technologies, and at the same 
time provides a road of long-range development for network and service providers. Under STL model, network providers will not only go on providing traditional voice communication service, but also provide more fundamental service, like multi-point switching, storage, speech translation, and so on. While the service providers will have more abilities from the network to create more new creative application for the society, then make the maximum value of the new communication network.

\section{Reference}

[1] D. Clark, "The Design Philosophy of the DARPA Internet Protocols", In Proceedings of ACM SIGCOMM 1988

[2] "Internet Performance Measurement and Analysis", http://www.merit.edu/ networkresearch/projecthistory/ipma/index.php? Printvs=1

[3] "IP Version 6 Working Group (ipv6)", http://www.ietf.org/html.charters/ipv6-charter.html

[4] “Advanced Networking for Leading-edge Research and Education”, http://abilene. Internet2.edu/

[5] "The GÉANT project", http://www.geant.net/

[6] “Asia-Pacific Advanced Network”, http://www.apan.net/

[7] "Next Generation Internet", http://www.ngi-net.de/

[8] "NewArch project: future-generation Internet architecture", http://www.isi.edu/newarch/

[9] D. Clark et al, "Tussle in Cyberspace: Defining Tomorrow's Internet", In Proceedings of ACM SIGCOMM 2002

[10] D. Clark et al, "Addressing Reality: An Architectural Response to Real-World Demands on the Evolving Internet”, In Proceedings of ACM SIGCOMM 2003 Workshops

[11] "FIND: future Internet network design," http://find.isi.edu

[12] "GENI: global environment for network innovations," http://www.geni.net

[13] "NSFCNET", http://www.nsfcnet.net/

[14] P. Francis, R. Gummadi, "IPNL: a NAT-extended Internet architecture," ACM SIGCOMM,'02 Aug 2002.

[15] M.Caesar, T. Condie, J. Kannan et al "ROFL: Routing on Flat Labels", ACM SIGCOMM'06, Aug 2006

[16] X. Yang, "NIRA: a new Internet routing architecture," SIGCOMM Workshop on Future Directions in Network Architecture (FDNA), Aug 2003.

[17] I. Stoica, D. Adkins, S. Zhuang, S. Shenker, S. Surana, "Internet indirection infrastructure," ACM SIGCOMM, Aug 2002.

[18] D. Cheriton, M. Gritter, “TRIAD: a scalable deployable NAT-based Internet architecture," Technical report, Jan 2000.

[19] Aibao Wang "The framework of content switch network oriented to the new generation Internet', 2004.9 Telecom Science 